\title{
Reporting for Duty: The duty cycle in Functional Electrical Stimulation research. Part I: Critical commentaries of the literature
}

\author{
Matthew J. Taylor (1,2), Ché Fornusek (3), Andrew J. Ruys (1) \\ (1) Faculty of Engineering and IT, University of Sydney, Camperdown, Australia; (2) Charles \\ Perkins Centre, University of Sydney, Camperdown, Australia, (3) Faculty of Medicine and \\ Health, University of Sydney, Lidcombe, Australia \\ This article is distributed under the terms of the Creative Commons Attribution Noncommercial License (CC BY-NC 4.0) which permits \\ any noncommercial use, distribution, and reproduction in any medium, provided the original author $(s)$ and source are credited.
}

\begin{abstract}
There are several parameters that can be modulated during electrical stimulation-induced muscle contraction to obtain external work, i.e., Functional Electrical Stimulation (FES). The literature has several reports of the relationships of parameters such as frequency, pulse width, amplitude and physiological or biomechanical outcomes (i.e., torque) when these parameters are changed. While these relationships are well-described, lesser known across the literature is how changing the duty cycle (time ON and time OFF) of stimulation affects the outcomes. This review provides an analysis of the literature pertaining to the duty cycle in electrical stimulation experiments. There are two distinct sections of this review - an introduction to the duty cycle and definitions from literature (part I); and contentions from the literature and proposed frameworks upon which duty cycle can be interpreted (part II). It is envisaged that the two reviews will highlight the importance of modulating the duty cycle in terms of muscle fatigue in mimicking physiological activities. The frameworks provided will ideally assist in unifying how researchers consider the duty cycle in electrical stimulation (ES) of muscles.
\end{abstract}

Key Words:functional electrical stimulation (FES), stimulation parameters, duty cycle, review Eur J Transl Myol 28 (4): 313-322, 2018

In 1981, Moreno-Aranda and Seireg ${ }^{1}$ released a series of papers investigating force responses arising due to various electrical stimulation regimes in both humans and canines. ${ }^{1-3}$ While providing rudimentary work in quantifying the responses of muscle to sinusoidal currents, one particular comment by the authors, relating to their stimulator design is of worthy interest: The stimulator should be able to produce a muscle contraction, maintain it for a certain time and then let the muscle relax for another period of time. ${ }^{1}$ Although a seemingly obvious proposition, this quote highlights an essential paradigm in functional electrical stimulation. In between electrically-evoked contractions, relaxation is paramount to preserve muscle structure and function. Ultimately, one important electrical parameter known as the duty cycle is important for controlling and describing muscle "ON" and "OFF" times. This has been defined in various ways across the literature: Table 1 lists some of those definitions.

\section{Selection of duty cycle in research and treatments}

It has been suggested that duty cycles which utilize a shorter period are more advantageous to use in a physical therapy scenario, to shorten treatment time for the individual. ${ }^{12}$ In addition, the authors go as far to say that if the period of ES is indeed too long, then this results in other components of treatment having to be removed due to time constraints. ${ }^{12}$ If one examines their protocols though, a counter-argument could possibly be made. The authors compared a 10/50/10 protocol (6 mins total), with a $10 / 10 / 10$ protocol ( 3 mins 20 s total). These time periods are not that considerably different. One could thus question Nelson and Cowling's ${ }^{12}$ proposition that indeed using a more fatiguing duty cycle is quicker - if there are only to be 10 contractions performed in the therapy time. In 1989, Selkowitz asserted in his paper on high frequency ES, that "duration of contractions" and "rest interval" were different across protocols, thereby implicating an early example of duty cycle variation between researchers. ${ }^{13}$ Indeed, as is the case for overall protocol variation, duty cycle is associated with much variance when compared across the current literature. This may be due to different methods of expressing times $\mathrm{ON}$ and $\mathrm{OFF}$ between authors (table 1) or the fact that various duty cycles might be employed for different clinical uses, for 


\section{Table 1. Definitions of the Duty Cycle from literature}

- Bajd and Munih: Duty cycle is measure of how long a pulse runs, and the break in-between pulses. ${ }^{4}$

- Carmick: Duty cycle ON time: total time (ratio), given as a percentage. ${ }^{5}$

- Smit et al.: Stimulation-rest ratio/interval. ${ }^{6}$

- Baker et al.: The duty cycle of electrical stimulation is a measure that quantifies stimulation time in terms of time $\mathrm{ON}$ and time OFF. It is usually expressed as either a percentage or ratio. ${ }^{7}$

- Starkey: Duty cycle is the time that the current is at "peak intensity" compared with the OFF time. ${ }^{8}$

- Dunlop: Duty cycle - work: rest ratio. ${ }^{9}$

- Deley et al.: Duty cycle - The total time to complete one on/off cycle. ${ }^{10}$

- Naeem et al.: ... active stimulation time of a cycle ... ${ }^{11}$

example in Baker et al. ${ }^{7}$ As with other stimulation parameters, insight may be drawn regarding the effect of different duty cycles on muscle responses by analyzing the literature on FES exercise. Hakansson and Hull indeed argue that there are two ways in which ON and OFF times have been examined, via "empirical" and computational means. ${ }^{14}$ Both are essential to aiding our understanding of the physiology of different FES duty cycles. Moreover, in light of the suggestion that the best duty cycle to use for the best treatment is yet to be elucidated, ${ }^{15}$ it is essential that the existing literature is surveyed and gaps in knowledge are identified, effectively justifying further exploration into the physiological effects of different duty cycles of stimulation. In addition, ON and OFF periods can be controlled in order to execute needed exercise in a timed fashion. In the study of Stein et al., ${ }^{16}$ the authors used a $40 \%$ duty cycle to conduct a fatigue test, on grounds of other authors which discusses how tibialis anterior is usually activated for that percentage of time in gait cycle. This is demonstrative of how duty cycles of stimulation may be manipulated in order to produce stimulation patterns that are in concert with the normal muscle activation pattern of exercises such as walking and cycling. The duty cycle of an electrical stimulation exercise regime is also imperative to select in the context of patient comfort. Indeed, Carmick discusses how essential it is to use appropriate ON and OFF times to allow children with cerebral palsy to become accommodated to electrical stimulation. ${ }^{5}$ The literature, on ES for cerebral palsy, ${ }^{5,17}$ is useful for understanding therapeutic uses of various duty cycles in a select patient cohort. Duty cycles may change in accordance with patient comfort (e.g., Carmick) ${ }^{17}$ over time as they habituate to the electrical stimulus. However, duty cycles can also be changed on a basis of muscle strength and fatigue progression. In Carmick's study, ${ }^{17}$ the increase from a 1:2.5 duty cycle to a $1: 1$ duty cycle is also reflective of how a more fatiguing duty cycle can be used after treatment with a less fatiguing one. More generally, Doucet and Griffin assert that ON/OFF times are important for both comfort and "force development", highlighting the importance of this parameter in the practical sense. ${ }^{18}$ There are other examples of changing duty cycle due to tolerances of stimulation as well. One recent event to occur in the FES community is the CYBATHLON, ${ }^{19}$ an event where among other competitions, an FES bike race is held. ${ }^{20}$ In this event, teams employ various strategies ${ }^{19}$ in an effort to win the race. Use of FES bikes during the race is a complex task, with several variables such as the technology, stimulation and physical preparation of the driver needing to be considered. ${ }^{21}$ What is interesting is the role of the duty cycle in preparing for the event. In a report by Guimarães et al., ${ }^{22}$ the authors discussed how duty cycle was changed from $1: 3$ to $1: 2$ to $1: 1$, when stimulating a pilot for pre-training. The authors discussed how duty cycle was increased based on the pilots' tolerability of exercise. In addition, Leung, et al. compared two forms of stimulation with one being more intermittent than the other in their study from the Chinese University of Hong Kong. ${ }^{23}$ Therefore, the duty cycle can be changed to increase intensity of exercise in FES research projects or competitive events such as a cycling race preparation. The duty cycle may also be harnessed to increase therapy efficacy over time. A higher duty cycle will generally result in more force generation and fatigue, so can be increased as the endurance of muscles increases over time (however contradicting literature, namely Matsunaga et al., ${ }^{24}$ is discussed later). Two examples of situations where this may occur are; a training study, which sees the effect of a protocol over a time period such as a few weeks, and a clinical treatment regime. Examples of both are:

- Training study. Faghri et al. ${ }^{25}$ conducted a study into FES of select shoulder muscles (deltoid, supraspinatus), in conjunction with physical therapy. The authors noted that duty cycle was adjusted throughout the study from 10 s ON 12 s OFF, to 30 s ON 2s OFF, over a $5 \mathrm{wk}$ period. The authors argue that the duty cycle was changed as the induced performance of the muscle was enhanced over time.

- Clinical regimen. In a neuromuscular electrical stimulation (NMES) textbook, Baker et al. ${ }^{7}$ presents a sample protocol for the treatment of wrist joint range of motion deficits. The protocol commences at 4s ON, 12s OFF before increasing to $6 \mathrm{~s}$ ON, 6s OFF. (i.e., begins at a duty cycle of $25 \%$ and increases to a duty cycle of $50 \%$ ). 


\section{Physiological Remarks}

The mechanisms underlying recovery and fatigue as related to $\mathrm{ON}$ and $\mathrm{OFF}$ times are important to understand in the design of protocols with potential clinical translation. In terms of practical applications, it has been argued that if OFF times are increased, so too must be the total time which the protocol runs for. ${ }^{7}$ However, ON and OFF time manipulation is also useful to control muscles from a metabolic point-of-view. Giat et al. ${ }^{26}$ discuss for example, the metabolic importance of recovery between muscle contractions. They note that the recovery period stalls reduction in metabolites, while at the same time giving the muscle enough time to restore metabolites such as phosphocreatine $(\mathrm{PCr})$ and adenosine triphosphate (ATP). ${ }^{26}$ Appropriate choice of OFF times of stimulation are essential should these important metabolites be restored, facilitating further multiple contractions. In addition, it is important that duty cycles are chosen to minimized fatigue, that would also facilitate such contractions. In a rather straightforward manner, it has been put forward by MorenoAranda and Seireg: In order to allow enough resting time for the muscle cells it is necessary to vary the duty cycle of the ON-OFF frequency. ${ }^{1}$ The obvious implication of this claim is that a muscle must be allowed ample rest should fatigue be attenuated, which could reduce future contractile efficacy. However, this resting period is essential to allow for the neuromuscular synapsis to have enough time to prepare for another delivery of acetylcholine, the messanger that trigger post-synaptic muscle plasmalemma and thus muscle contraction. "Preparation" in this context refers to allowing the natural refractory period of the nerve to pass before another contraction is to take place. Moreno-Aranda and Seireg ${ }^{1}$ stipulate, indeed that rest periods should be at least $5 \mathrm{~ms}$ in length, as motor neurons and skeletal muscle cells have a 4-5 ms refractory period. By "rest period", one could assume this comment is referring to the interpulse interval (IPI) as $5 \mathrm{~ms}$ is too quick for a normal OFF segment in a duty cycle. Explanation of the importance of relaxation times as related to the refractory period may be highlighted be examining studies which have been conducted on various "rest" periods between muscle contractions. It has been shown for example, that the maximum force and rate of force development of rat soleus and extensor digitorum longus after a bout of 30 tetanic contractions changes differently depending on the intertetanus interval (i.e., OFF time) ${ }^{27}$ If stimulation is carried out using smaller OFF times between contractions for example, there is an increase in fatigue. ${ }^{28}$ In a peripheral fatigue context, one reason which may explain this increased fatigue is that the refractory period of axons increases according to fatigue. ${ }^{29}$ Therefore, training at the same duty cycle will result in increase in fatigue if muscles are not given ample time to relax between pulse trains. There is a range of other metabolic results reported in the literature on duty cycle. In a study by Ko and Joung for example, ${ }^{30}$ "blood lactate rate" in a group of 20 Korean house rabbits was much higher following twenty minutes of stimulation at a $20 \%$ duty cycle, as opposed to a $50 \%$ one (as an aside, this is interesting as would not lactate be greater for $50 \%$ because of more muscle fatigue?). Therefore, the choice of duty cycle may have an effect on acid metabolites accumulation in muscle. In addition, the duty cycle may also have an effect on tissue oxygenation, as stimulation ON/OFF times dictate the relative amount of contraction and relaxation that will occur. Takuma and Shimada for example, ${ }^{31}$ argue that in spite of relaxation times between muscle contractions in intermittent isometric exercise, over time there can be increasing levels of deoxygenation. The authors assert that anaerobic metabolism is utilized due to the occlusion of blood flow which occurs during isometric exercise. ${ }^{31}$ Consequentially, the duty cycle is also a paramount consideration in determining what energy store is used. Shorter relaxation times ensue due to augmentation of fatigue, ${ }^{28}$ and following on from the comments of Takuma and Shimada, ${ }^{31}$ it would seem that this could also accelerate the relative switch from aerobic to anaerobic metabolism.

\section{State of the Art: Duty Cycle studies of pulsed current}

Following on from the work of Liberson et al. ${ }^{32}$ on footdrop stimulation, the plethora of papers on functional electrical stimulation have investigated several aspects of ES treatment - stimulation parameter studies an important example. Some authors have focused on the effects of different duty cycles on muscle force generation, and this is often assessed by examining decrease in torque or force over time. ${ }^{33-36}$ It should be noted also that some studies express their aim rather as to investigate the effect of relaxation time on fatigue, for example Duchateau and Hainaut, ${ }^{37}$ or continuous versus intermittent stimulation, for example Spriet et al. ${ }^{34}$ In the context of this paper, both are related to understanding the effect of duty cycle modulation on fatigue. Select literature pertaining to duty cycle investigations is presented in the following Table 2. For the purposes of orderly discussion, studies have been listed in chronological order. Annotations for Table 2 are listed in the successive Table 3.

Table 2. Select Pulsed Current Studies of Duty Cycle [Parameters, Some Conclusions] and Critical Commentaries (in [ ])

\section{Study Summaries}

- Eriksson and Häggmark ${ }^{38}$ used a $5-6$ s ON 5s OFF (i.e., rest) for $1 \mathrm{hr} / \mathrm{d}, 5 \mathrm{~d} / \mathrm{wk}, 4 \mathrm{wk}$ in patients who underwent ligament surgery for the knee. Other relevant stimulation parameters include: $200 \mathrm{~Hz}^{*}$, $100 \mathrm{~V}$ or less (this was the value for the "pain threshold"). They compared enzymatic activity of succinate dehydrogenase (SDH) across two groups - 
plaster-cast and "isometric training" versus the same but with percutaneous stimulation as well. The authors concluded that the group who received ES of femoral nerve had a greater SDH activity than those who did not.

- Godfrey et al., as cited by Packman-Braun, ${ }^{39}$ argued 1:5 best for holding force above $50 \%$ initial value. They put forward that 1:6, 1:10 could even be better than $1: 5 * *$.

- Benton and Montgomery presented data from dorsiflexors subjected to $1 \mathrm{~s}$ ON 1s OFF, 1s ON $2 \mathrm{~s}$ OFF, $1 \mathrm{~s} \mathrm{ON} \mathrm{5s} \mathrm{OFF.}{ }^{40}$ As relaxation time increases, "decrease in percent maximum force" is less severe. However, there are several points to be made regarding this dataset from a critical point of view. One is: it is unclear how many subjects were subjected to the different duty cycle protocols.

- Duchateau and Hainaut studied the effect of various rest periods on fatigue in healthy adductor pollicis. ${ }^{37}$ Stimulation was provided in bouts of $60 \mathrm{~s}$ ("sustained"; 1s ON 2s OFF, 1s ON 1s OFF, 1s ON $0.5 \mathrm{~s}$ OFF). Other relevant parameters were $30 \mathrm{~Hz}$, $0.1 \mathrm{~ms}$. The authors found as the rest interval between contractions increased, the decline in initial force after $60 \mathrm{~s}$ decreased. [In the first $30 \mathrm{~s}$, decline in percentage of initial force was very close for all three duty cycles (i.e. rest periods of $2 \mathrm{~s}, 1 \mathrm{~s}, 0.5 \mathrm{~s}$ ). One possible implication of this is that if we stimulate with any of these relaxation periods for a 30 s period, that they will lead to similar decline in force and hence fatigue. Hence, 30s protocols (spaced out with ample rest periods), of different combinations of these rest periods could be used for optimization/training purposes (i.e., see what combos yield the least decrease in force over several sets of 30 s stimulation periods)].

- Cox et al. ${ }^{41}$ examined how torque decreased when the quadriceps was stimulated with three different duty cycles over 10 contractions $(10: 35,10: 50,10: 65$ in $\mathrm{s}$ domain)***. They performed these experiments using two different electrode arrangements (lumbosacral plexus, and motor point). Stimulation was done at 100 $\mathrm{Hz},-60$ degrees knee joint extension, $1 \mathrm{~ms}$. The authors found that for the 50 and $65 \mathrm{~s}$ rest periods, reduction in torque was similar. Most torque metrics also decreased greater than 50 and 65 for the 35 s rest period as well.

- Krajl et al. ${ }^{42 * * * *}$ present a series of fatigue curves comparing continuous ES with cyclical (i.e., intermittent). Stimulation was given to the quadriceps at $20 \mathrm{~Hz}, 300 \mu \mathrm{s}$, rectangular. Results were presented for an individual "after 42 days of exercising". The authors present a range of different fatigue profiles for quadriceps subjected to a range of different duty cycles. They argue that cyclical stimulation is effective for attenuating fatigue, relative to a continuous stimulation pattern. The authors discuss how their "...measurements suggest that duty cycles with ON time of 1,2, or 3 times greater compared to the OFF time will fatigue the muscle in a manner similar to continuous activation". At a glance of their graph though, other interesting observations may be elucidated, namely:

o Different "multiples" of a 1:1 duty cycle have different decreases in initial torque. Their graphs show 1s ON 1s OFF < 8s ON 8s OFF < 4s ON 4s $\mathrm{OFF}$ in the context of fatigue resistance [origin of data uncertain].

o It would also be expected that as relaxation time between ON segments increases, fatigue would decrease. Yet, their graph of five minutes stimulation [has some potentiation occurred?] shows a series of profiles for $4 \mathrm{~s}$ ON with different relaxation times $(4 \mathrm{~s}$, 8 s, 10s) (i.e., corresponding to $1: 1,1: 2,1: 2.5)$. The $1: 1$ duty cycle is most fatiguing, as inferred by manual inspection. 1:2.5 is less fatiguing for various parts of the graph, which seems intuitive due to the increased rest time. Yet - at some points the 1:1 and 1:2.5 seems to be producing similar force as seen by examining the graph (not statistical significance). In addition, the $4 \mathrm{~s}$ ON $8 \mathrm{~s}$ OFF (i.e. 1:2) seems less fatiguing than the $4 \mathrm{~s}$ ON 10s OFF (i.e., 1:2.5) which is peculiar as the latter has a longer rest period so one would assume there would be greater recovery of force as there has been more time for metabolites such as ATP to collect within the muscle (e.g., Giat et al., 1996) ${ }^{26}$, facilitating subsequent contractile activity.

- Chasiotis et al. ${ }^{43}$ suggest that intermittent can cause greater decrease in force than continuous, in their study of healthy quadriceps $(n=4)$. Stimulation was delivered over a short period of time.

- Cole et al. ${ }^{44}$ [abstract] in a similar fashion to Gentz and Moore, ${ }^{36}$ examined the fatigue arising from ES isometric with various duty cycles over a 30min period $(\mathrm{n}=10$ female). They examined various cycles at $50 \mathrm{~Hz}(1: 3,1: 5,1: 7,1: 10)$ and also one at $30 \mathrm{~Hz}$ $(1: 3) . * * * * *$ Torque at the commencement of the tests was " $20+4 \mathrm{ft}-\mathrm{lbs}$ " + Results of these studies were:

o For the $50 \mathrm{~Hz}$ data, as relaxation time increased, the authors noted the times where "statistically significant decreases in torque output" occurred. One would expect as relaxation times increases, so too would this value [meaning of stat. sig. decrease in torque output is unclear].

o Authors also found at $\mathrm{t}=30 \mathrm{mins}$, there were stat sig diffs: * $1: 3(50 \mathrm{~Hz})$ vs $1: 7,1: 10(50 \mathrm{~Hz}), 1: 3$ $(30 \mathrm{~Hz})$ [This shows that if a $1: 3$ duty cycle is delivered at different frequencies there will be a stat sig diff in torque output after 30mins? Or torque decline?]. Authors stipulated 1:5 had similar relationship. 
o $1: 3(30 \mathrm{~Hz})$ compared with $1: 7(50 \mathrm{~Hz}), 1: 10(50 \mathrm{~Hz})$ - not statistically different.

o Authors argue 1:10 good for treatment on grounds of less fatigue.

- Bergström and Hultman investigated continuous and intermittent contractions of healthy vastus lateralis. ${ }^{35}$ They examined a duty cycle of $1: 1$ for periods of $0.8 \mathrm{~s}$ and 3.2s. Stimulation was delivered at $300 \mu \mathrm{s}, 20 \mathrm{~Hz}$. Voltage changed such that $25 \%$ was generated by the muscle contractions. Experiments were conducted such that the 0.8 s protocol was conducted on one leg, and the $3.2 \mathrm{~s}$ protocol on the other leg. Both legs received a total contraction time of 51.2s. The authors concluded that a continuous stimulation protocol++ caused the least decay in force (i.e., percentage of initial force), in comparison with $0.8 \mathrm{~s}$ and $3.2 \mathrm{~s}$ protocols. [It is peculiar that the authors concluded that shorter periods of electrical stimulation resulted in more force decline. One would think that a continuous protocol would be more fatiguing, and thus cause the largest decrease in force. Perhaps this is because the data analysed was over a short period of time before fatigue becomes deleterious to useful force generation].

- Gentz and Moore [abstract] ${ }^{36}$ examined the effects of stimulation at a 1:3 duty cycle on quadriceps torque reduction over time. They performed stimulation at $30 \mathrm{~Hz}$, for $30 \mathrm{mins}$ isometric quadriceps. They used various "multiples" of the $1: 3$ cycle $(1: 3,2: 6,4: 12$, $8: 24,12: 36)$. Initial torque was set to be $20+/-1 \mathrm{ft} . \mathrm{lbs}$ (i.e. $27.1+/-1.4 \mathrm{Nm}$ ) [Converted with assistance of Ref:http://www.convertunits.com/from/ft+lb/to/N+m, accessed 1/2/16]. Interestingly, they found decreases in "torque output" occurred at 4 mins (for 1:3, 4:12, 12:36), 6 mins (2:6), and 8 mins (8:24). While increasing $\mathrm{ON}$ times usually leads to a quicker fatigue (i.e. reduction in force or torque), this study seems to be in discordance with this theory. One would expect that from 1:3 to 12:36, that decrease in torque would occur much quicker as the $\mathrm{ON}$ time is increased. This was clearly not the case.

o In addition to the aforementioned disparity, these results also illustrate an important point. For a given duty cycle, multiples of the same cycle can cause different torque responses. [Yet they say below stat sig.].[Note: Important. Authors argue "no sig diff" between 1:3, 2:6, 4:12, 8:24. They say these should be chosen over 12:36 though to reduce fatigue. But the differences above.... are they important?].

- Packman-Braun ${ }^{45}$ looked at the fatigue resulting from ES of wrist extensors, $300 \mu \mathrm{s}$ PW, 36 pps. Stimulation was done at 1:1, 1:3, 1:5. The defined ON as 2 s of rest, $5 \mathrm{~s} \mathrm{ON}$, and ratios were relative to the $5 \mathrm{~s}$ value (i.e. $5 \mathrm{~s} \max \mathrm{ON}, 5,15$ or $25 \mathrm{~s}$ OFF). The authors provided stimulation until force fell below $50 \%$ of initial force, or for a time of $30 \mathrm{mins}$. Force recordings from wrist joint extension was carried out using a strain gauge. They argue that the lower the rest period, the more fatigue, yet there could be some contention surrounding these findings.+++

- Pournezam et al..$^{46}$ looked at changes in knee moment after fatigue and recovery, for three of the quadriceps $(\mathrm{RF}, \mathrm{VL}, \mathrm{VM})$. The cohort examined were SCI $(\mathrm{n}=$ 2). The study was aimed at comparing two modalities of stimulation - "sequential" (one muscle at a time) or "continuous". Other relevant parameters were:

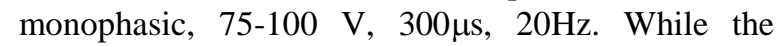
primary impetus of this work was not to examine duty cycles per se, the findings of this paper are applicable to duty cycle and quadriceps stimulation. For example, the authors compared "three-phase" ++++, +++++ sequential stimulation (i.e., each of the RF, VL, VM in succession - "33\% duty cycle per muscle"), with continuous stimulation in terms of time taken for knee moment to be reduced to $50 \%$ of maximum. With the continuous protocol, all three muscles elicited a KJM of $50 \%$ by $63 \mathrm{~s}$ or less. However with the sequential protocol, time taken to reach this value was 7 mins. Hence this shows that a sequential (i.e., intermittent) is much less fatiguing than a continuous protocol.

- Snyder-Mackler et al ${ }^{47}$ studied ES triceps at three duty cycles in the .10s domain: 1:3, 1:5, 1:7, to look at fatigue. The found:

o Greatest fatigue at 1:3 after 15 mins.

o Greatest time to reach half of initial force was 1:7.

o No diffs between all 3 DC's after 10, 20, 30 contractions.

o The authors argue that "...absolute number of contractions..." are the important factor in fatigue.

- Snyder-Mackler et al. [1988b] $]^{48}$ studied ES triceps at three duty cycles in the .10 s domain: 1:3, 1:5, 1:7, to look at strength. $6 \mathrm{hr} / \mathrm{d}, 5 \mathrm{~d} / \mathrm{wk}, 3 \mathrm{wk}$. No sig diffs in MVIC's after, the authors concluded inadequate for strength increases.

- Stein et al. ${ }^{16}$ conducted a study on tibialis anterior over 30wk (different times of stimulation each day 5 different protocols for $6 \mathrm{wk}$ each). Stimulation was provided at $20 \mathrm{~Hz}$ ("stimuli/s"), 5s ON, 5s OFF (50\%).

- A comprehensive study by Lieber and Kelly examined knee joint extension torques in a sample of 90 individuals. ${ }^{49}$ The authors used six combinations of stimulation sequences, testing $50 \%$ and $70 \%$ duty cycles of stimulation for each of the frequencies 10 , 30 or $50 \mathrm{~Hz}$, arguing that these were reflective of what is used in a clinical context^^${ }^{\wedge}$. Stimulation was delivered for 30mins - different number of contractions for different duty cycles ${ }^{\wedge}$. However, there were differences in average torque, with the greatest being produced by the $50 \mathrm{~Hz}$ stimulation at a duty cycle of $50 \%$. 
- Nelson and Cowling state that the most often used duty cycle is $1: 5(10 / 50 / 10)^{\wedge \wedge \wedge}$, in the context of muscle strengthening. ${ }^{12}$ The authors compared strength gains in healthy quadriceps between a 1:1 and 1:5 duty cycle. The authors measured MVIC before and after a training period of $4 \mathrm{wk}$ (3 sessions per wk). Other parameters of relevance were: $200 \mu \mathrm{s}$, $50 \mathrm{~Hz}$. Training was done in each session with 10 isometric contractions, at a stimulation level that could be "maximally tolerated" by each subject. They found a $13 \%$ change in MVIC of 1:1 group, and a $10 \%$ change in MVIC of 1:5 group ${ }^{\wedge \wedge \wedge \wedge}$. The authors argue that there was no significant difference thus between each protocol, in terms of "strength gains". The authors argue on a basis of their findings, that time of treatment may be reduced by implementing a

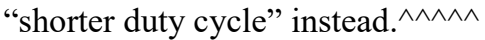

- Correa et al. ${ }^{50 \$}$ found no difference in their parameter of interest as related to the duty cycle of electrical stimulation. Blood lactate resorption was found to be the same, when tibialis anterior was stimulated at $50 \mathrm{~Hz}$, using duty cycles of $10 \mathrm{~s}$ ON, $10 \mathrm{~s}$ OFF and $10 \mathrm{~s}$ ON, 30s OFF.

- Hakansson and Hull ${ }^{14}$ point to other authors $\$$ : That a DC of $20 \%$ (i.e. 1:5) is preferential in the context of muscle endurance than higher duty cycles. [This could also be used as evidence that "optimal" duty cycles change depending on the exercise in question]. Their paper is on ES-cycling.

- Marion et al. ${ }^{51}$ looked at stimulation of quadriceps in healthy individuals. Did ISO at four knee angles (15, 40, 65, 90 degrees). $600 \mu \mathrm{s}, 1 \mathrm{~s}$ ON 10s OFF for trains. $4 \mathrm{~min}$ rest between each angle of testing.

- Naeem et al. ${ }^{11}$ studied the effects of low frequency stimulation of the biceps brachii muscle at various duty cycles. The authors trialled each of 5 and $10 \mathrm{~Hz}$, at duty cycles of $50 \%$ and $85 \%, 100 \mathrm{~mA}$.

o Duty cycles. $85 \%$ lower than $50 \%$ initially. [Cause of this uncertain].

o Study design. [Only over at $1 \mathrm{~min}$ time period ... so interpretation is difficult. The authors of this review could repeat this study over a much longer period of time should it have practical implications for the field of FES].

- Sillen et al. ${ }^{15}$ reviewing 18 NMES trials noted that across these studies, duty cycle was reported in the range $3 \mathrm{~s}-55 \mathrm{~s}$ ON, $30 \mathrm{~s}-2 \mathrm{~s}$ OFF.

Annotations for Table 2 are listed below in Table 3.

Table 3. Salient Commentaries on Table 2 Literature

*The authors argue that their protocol allowed for tetanic contractions. Yet, fusion can occur at a much lower frequency than $200 \mathrm{~Hz}$.
**[Regarding Baker and colleagues]: Commentary on a related paper. Baker et al. ${ }^{7}$ Do the authors refer to fatigue? Baker et al. argued at the time of their publication that how long a contraction goes for, is just as imperative to understand as are ON/OFF ratios. For example, [do they say the next comment on a basis of refs 6 and 136?]: the authors note that while a duty cycle of $1: 3$, in the $4 \mathrm{~s}$ domain may be appropriate, $1: 3$ in the 12 s may not be acceptable ("... is generally too high...”). Why? For fatigue? Or resting time too high in between contractions?

***Perhaps a useful experiment would be for one to repeat the work of Cox et al. ${ }^{41}$ over a much longer period of time. Then, long-term torque decreases could be ascertained for each of the rest intervals analysed.

****It should be noted that these observations from the work of Krajl et al. ${ }^{42}$ : a) May be from FES standing data, b) are based on observation, rather than statistical comparisons of duty cycle profiles over time, c) are from a case study, $\mathrm{n}=1$. Evidently such observations suggest that there is more work to be done in this field to clarify the relative effects of different duty cycles of stimulation on torque responses.

$* * * * *$ The authors argue that $1: 3$ at $30 \mathrm{~Hz}$ was used "...for standardization" purposes. Meaning of this is unclear.

+ Should this be a $+/$ - instead, in their paper?

++ The continuous data they used for comparative purposes [they refer to reference 5] was taken from a study which used a different sample size to their work. This could potentially have impact on the conclusions drawn from the results?

+++ The general trend of Packman-Braun's data is that a $1: 1$ results in most patients reaching $<50 \%$ quickly, whereas with a duty cycle of $1: 5$, this occurs later on. ${ }^{45}$ 1:3 mixed though; so perhaps investigating intermediate DC's warrants further results. Too general to propose that as the duty cycle increases, force time also does.

++++ Duty cycle defined relative to the continuous protocol.

+++++Three-phase was a permutation of stimulation between the three muscles RF, VL, VM for one-third of the total time. The authors also conducted a two-phase permutation with RF ON for half the time, VL+VM ON for the other half of the time.

${ }^{\wedge}$ Other authors don't justify their choice of parameters, so this is a comprehensive comment by the authors.

$\wedge^{\wedge}$ The authors found no differences in torque/time integral across groups. What does this physiologically represent?

${ }^{\wedge \wedge}$ The whole "10/50/10" notation may have become common in the literature following the work of authors such as Selkowitz ${ }^{13}$ and further stemming from early work by Russian Kots ${ }^{52}$ whom Selkowitz discusses. Nelson and Cowling used pulsed current with this 
notation. ${ }^{12}$ However, if this notation is used for high frequency alternating current studies (e.g., the early Russian studies), then how can comparisons be made between such studies if the same notation is used for stimulation patterns which differ in frequency by a factor of $102-103$ ?

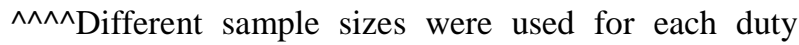
cycle $(1: 1, n=13 ; 1: 5, n=7)$. Perhaps a more sound methodology would use; a) the same sample size for each group, or b) both protocols on each subject, one on one leg, one on the other (e.g., Bergström and Hultman). ${ }^{35}$

$\wedge \wedge \wedge \wedge \wedge$ If a similar experiment was conducted, where more than 10 isometric contractions were performed, one would hypothesize that a 1:1 duty cycle would be undesirable to use because of significant muscle fatigue over time.

\$The author of this paper was only able to discern this information from the abstract, as translation would be requisite for a full understanding of the authors' methodology.

\$Namely, references 19, 57 and 58.

The duty cycle has also been looked at as a parameter of interest in studies of pressure sore attenuation, and select studies from the literature will be presented in table 5 [of Part II of this review]. ${ }^{53}$

\section{Concluding Remarks of Part I}

It is clear that duty cycle definitions vary across the literature. In addition, the relative ability of intermittent and continuous stimulation to fatigue muscle is, at best, contentious across literature. In following from the literature and concepts discussed in this Review Part I, ${ }^{54}$ we will propose a series of frameworks to help unify duty cycle analysis in stimulation protocols. These will be explored extensively in the Part II of this review. ${ }^{53}$ We are confident that our suggestions will be followed in the training of those thoracic-level SCI persons after their enrollment in the future studies on intrethecal approach of FES. ${ }^{55-67}$

\section{List of acronyms}

ATP - adenosine triphosphate

DC - duty cycle

ES - Electrical stimulation

FES - Functional Electrical Stimulation

$\mathrm{Hz}-\mathrm{Hertz}$

IPI - inter pulse interval

ISO - isometric

$\mathrm{KJM}$ - knee joint moment

MVIC - maximal voluntary isometric contraction

NMES - neuromuscular electrical stimulation

$\mathrm{PCr}$ - phosphocreatine

Pps - pulses per second

PW - pulse width

$\mathrm{RF}$ - rectus femoris
SDH - succinate dehydrogenase

$\mathrm{V}-$ volts

$\mathrm{VL}$ - vastus lateralis

$\mathrm{VM}$ - vastus medialis

\section{Author's contributions}

MJT drafted the manuscript, was responsible for literature review and critical synthesis and analysis of literature, $\mathrm{CF}$ and AJR gave feedback on the manuscript.

\section{Acknowledgments}

MJT is a PhD student at the University of Sydney, Australia. He wish to acknowledge Professor Ugo Carraro, M.D. for his advice on structuring of the manuscript.

Funding: None.

\section{Conflict of Interest}

The authors declare no conflicts of interests.

\section{Ethical Publication Statement}

We confirm that we have read the Journal's position on issues involved in ethical publication and affirm that this report is consistent with those guidelines.

\section{Corresponding Author}

Mr. Matthew James Taylor, Level 3 East, Charles Perkins Centre D17, The University of Sydney, NSW, 2006, Australia. Phone: + 6128627 0521. Mobile: + 61 478033 859. Email: matthew.j.taylor@ sydney.edu.au

\section{E-mail of co-authors}

Ché Fornusek: che.fornusek@sydney.edu.au Andrew J. Ruys: andrew.ruys@sydney.edu.au

\section{References}

1. Moreno-Aranda J, Seireg A. Electrical parameters for over-the-skin muscle stimulation. J Biomech 1981;14:579-85.

2. Moreno-Aranda J, Seireg A. Investigation of overthe-skin electrical stimulation parameters for different normal muscles and subjects. J Biomech 1981;14:587-93.

3. Moreno-Aranda J, Seireg A. Force response to electrical stimulation of canine skeletal muscles. J Biomech 1981;14:595-9.

4. Bajd T, Munih M. Basic functional electrical stimulation (FES) of extremities: An engineer's view. Technol Health Care 2010;18:361-9.

5. Carmick J. Guidelines for the clinical application of neuromuscular electrical stimulation (NMES) for children with cerebral palsy. Pediatr Phys Ther 1997;9:128-36.

6. Smit CAJ, Legemate KJA, de Koning A, et al. Prolonged electrical stimulation-induced gluteal and hamstring muscle activation and sitting pressure in spinal cord injury: Effect of duty cycle. J Rehabil Res Dev 2013;50:1035-45. 
7. Baker LL, Wederich CL, McNeal DR, et al. Neuro Muscular Electrical Stimulation - A Practical Guide $\left(4^{\text {th }}\right.$ ed). Rehabilitation Engineering Program, Los Amigos Research \& Education Institute, Rancho Los Amigos National Rehabilitation Centre, United States of America; 2000.

8. Starkey C. Therapeutic modalities for athletic trainers. F.A. Davis Company, Philadelphia, United States of America; 1993.

9. Dunlop RJ. Electrical muscle stimulation protocols: Effects on force production and energy metabolism of the gastrocnemius muscle in humans. Master of Physical Education Thesis, The Faculty of Graduate Studies, The University of British Columbia; 1991.

10. Deley G, Denuziller J, Babault N. Functional electrical stimulation: cardiorespiratory adaptations and applications for training in paraplegia. Sports Med 2015;45:71-82.

11. Naeem J, Azman AW, Khan S, Mustafah YM. An investigation of fatigue phenomenon in the upper limb muscle due to short duration pulses in an FES system. $5^{\text {th }} \quad$ International Conference on Mechatronics (ICOM '13), 2-4 July, 2013, Kuala Lumpur, Malaysia. IOP Conference Series: Materials Science and Engineering.

12. Nelson BE, Cowling AE. Effect of duty cycle on quadriceps femoris muscle isometric strength gains with electrical stimulation. School of Physical Therapy, paper 176. Master of Science in Physical Therapy Thesis, School of Physical Therapy, Pacific University, Forest Grove, Oregon, United States of America; 1999.

13. Selkowitz DM. High frequency electrical stimulation in muscle strengthening - A review and discussion. Am J Sports Med 1989;17:103-11.

14. Hakansson NA, Hull ML. Muscle stimulation waveform timing patterns for upper and lower leg muscle groups to increase muscular endurance in functional electrical stimulation pedaling using a forward dynamic model. IEEE Trans Biomed Eng 2009;56:2263-70.

15. Sillen MJH, Franssen FME, Gosker HR, et al. Metabolic and structural changes in lower-limb skeletal muscle following neuromuscular electrical stimulation: A systematic review. PLoS ONE 2013;8:e69391.

16. Stein RB, Gordon T, Jefferson J, et al. Optimal stimulation of paralyzed muscle after human spinal cord injury. J Appl Physiol 1992;72:1393-1400.

17. Carmick J. Clinical use of neuromuscular electrical stimulation for children with cerebral palsy, Part 1: Lower extremity. Phys Ther 1993;73:505-13.

18. Doucet BM, Griffin L. High-versus low-frequency stimulation effects on fine motor control in chronic hemiplegia: A pilot study. Top Stroke Rehabil 2013;20:299-307.

19. Coste CA, Bergeron V, Berkelmans R, et al. Comparison of strategies and performance of functional electrical stimulation cycling in spinal cord injury pilots for competition in the first ever CYBATHLON. Eur J Transl Myol 2017;27(4):7219. doi: 10.4081/ejtm.2017.7219. eCollection 2017 Dec 5.

20. Cybathlon. http://www.cybathlon.ethz.ch/discipline s/fes-race.html. Accessed 25/7/18.

21. Berkelmans R, Woods B. Strategies and performances of Functional Electrical Stimulation Cycling using the BerkelBike with Spinal Cord Injury in a competition context (CYBATHLON). Eur J Transl Myol 2017;27(4):7189. doi: 10.4081/ejtm.2017.7189. eCollection 2017 Dec 5.

22. Guimarães JA, da Fonseca LO, de Sousa AC, et al. FES Bike Race preparation to Cybathlon 2016 by EMA team: a short case report. Eur J Transl Myol 2017;27(4):7169. doi: 10.4081/ejtm.2017.7169. eCollection 2017 Dec 5.

23. Leung KW, Tong RK, Wang $\mathrm{X}$, et al. The Effectiveness of Functional Electrical Stimulation (FES) in On-Off Mode for Enhancing the Cycling Performance of Team Phoenix at 2016 Cybathlon. Eur J Transl Myol 2017;27(4):7132. doi: 10.4081/ejtm.2017.7132. eCollection 2017 Dec 5.

24. Matsunaga, T, Shimada, Y, Sato, K. Muscle fatigue from intermittent stimulation with low and high frequency electrical pulses. Arch Phys Med Rehabil 1999;80:48-53.

25. Faghri, PD, Rodgers, MM, Glaser, RM, et al. The effects of functional electrical stimulation on shoulder subluxation, arm function recovery, and shoulder pain in hemiplegic stroke patients. Arch Phys Med Rehabil 1994;75:73-79.

26. Giat, Y, Mizrahi, J, Levy, M. A model of fatigue and recovery in paraplegic's quadriceps muscle subjected to intermittent FES. J Biomech Eng 1996;118:357-366.

27. Barclay, CJ. Effect of fatigue on rate of isometric force development in mouse fast- and slow-twitch muscles. Amer J Physiol 1992b;263(Cell Physiol 32):C1065-C1072.

28. Binder-Macleod, SA, Snyder-Mackler, L. Muscle Fatigue: Clinical implications for fatigue assessment and neuromuscular electrical stimulation. Phys Ther 1993;73(12):83-91.

29. Borg, J, Grimby, L, Hannerz, J. The fatigue of voluntary contraction and the peripheral electrical propagation of single motor units in man. J Physiol 1983;340:435-444.

30. Ko, T, Joung, H. The effects of changing duty cycle with electrical stimulation on blood lactate and plasma enzyme. Phys Ther Korea 2005;12(2):9097. [Abstract in English, Paper in Korean].

31. Takuma Y, Shimada T. Effect of duration of muscle relaxation during intermittent isometric exercises on deoxygenation and lactate accumulation in active muscles. J Phys Ther Sci 2011;23:495-501. 
32. Liberson WT, Holmquest HJ, Scot D, Dow M. Functional electrotherapy: Stimulation of the peroneal nerve synchronized with the swing phase of the gait of hemiplegic patients. Arch Phys Med Rehabil 1961;42:101-5.

33. Barclay CJ, Loiselle DS. Dependence of muscle fatigue on stimulation protocol: effect of hypocaloric diet. J Appl Physiol 1992;72:2278-84.

34. Spriet LL, Soderlund K, Hultman E. Energy cost and metabolic regulation during intermittent and continuous tetanic contractions in human skeletal muscle. Can J Physiol Pharmacol 1988;66:134-9.

35. Bergström M, Hultman E. Energy cost and fatigue during intermittent electrical stimulation of human skeletal muscle. J Appl Physiol 1988;65:1500-5.

36. Gentz L, Moore C. Effect of duty cycle on fatigue during stimulation of the quadriceps muscle. Phys Ther 1988;834-834 [Abstract R-287].

37. Duchateau J, Hainaut K. Electrical and mechanical failures during sustained and intermittent contractions in humans. J Appl Physiol 1985;58:942-7.

38. Eriksson E, Häggmark T. Comparison of isometric muscle training and electrical stimulation supplementing isometric muscle training in the recovery after major knee ligament surgery. Am J Sports Med 1979;7:169-71.

39. Godfrey CM, Jayawardena H, Quance TA, et al. Comparison of electrostimulation and isometric exercise in strengthening the quadriceps muscle. Physiotherapy Canada 1979;31:365-7.

40. Benton LA, Montgomery J. Functional electrical stimulation: A practical clinical guide ( $2^{\text {nd }}$ ed.) Professional Staff Association, Rancho Los Amigos Rehabilitation Engineering Center, Rancho Los Amigos Hospital; Downey CA:31-52;1981.

41. Cox AM, Mendryk SW, Kramer JF, Hunka SM. Effect of electrode placement and rest interval between contractions on isometric knee extension torques induced by electrical stimulation at $100 \mathrm{~Hz}$. Physiotherapy Canada 1986;38:20-7.

42. Krajl A, Bajd T, Turk R, Benko H. Posture switching for prolonging functional electrical stimulation standing in paraplegic patients. Paraplegia 1986;24:221-30.

43. Chasiotis D, Bergström M, Hultman E. ATP utilization and force during intermittent and continuous muscle contraction. J Appl Physiol 1987;63:167-74.

44. Cole KE, Sledge MM, Baker LL, McNeal DR. Muscle fatigue during electrically induced isometric contractions at varying duty cycles. Phys Ther 1987;67:792.

45. Packman-Braun R. Relationship between functional electrical stimulation duty cycle and fatigue in wrist extensor muscles of patients with hemiparesis. Phys Ther 1988;68:51-6.
46. Pournezam M, Andrews BJ, Baxendale RH, et al. Reduction of muscle fatigue in man by cyclical stimulation. J Biomed Eng 1988;10:196-200.

47. Snyder-Mackler L, Campbell L, Kenney ME, et al. Effect of duty-cycle of portable neuromuscular electrical stimulation on fatigue of non-dominant triceps. Phys Ther 1988;68:833[Abstract R-283].

48. Snyder-Mackler L, Cellucci M, Magno J, et al. Effect of duty cycle of portable neuromuscular electrical stimulation on strength of non-dominant triceps. Phys Ther 1988;68:833[Abstract R-284].

49. Lieber RL, Kelly ML. Torque history of electrically stimulated human quadriceps: Implications for stimulation therapy. J Orthop Res 1993;11:131-41.

50. Corrêa FI, Corrêa JCF, Tessarolo AdA, et al. Avaliação do ácido lático em indivíduos com hemiparesia pós-acidente vascular encefálico após estimulação elétrica para fortalecimento muscular. [Lactic acid assessment in post-stroke hemiparetic subjects following electrical stimulation for muscle strengthening]. Fisioterapia e Pesquisa, São Paulo 2009;16(2):178-82. [Paper in Portuguese, Abstract in Portuguese and English].

51. Marion MS, Wexler AS, Hull ML. Predicting nonisometric fatigue induced by electrical stimulation pulse trains as a function of pulse duration. J Neuroeng Rehabil 2013;10:13.

52. Kots YM. Electrostimulation of skeletal muscles: Canadian-Soviet exchange symposium: Lecture notes. Concordia University, December 6-15, 1977. Translated into English by Babkin and Timtsenko.

53. Taylor MJ, Fornusek C, Ruys AJ. The duty cycle in Functional Electrical Stimulation (FES) research Part II: Duty cycle multiplicity and domain reporting. Eur J Transl Myol 2018;28: In press

54. Taylor MJ, Fornusek C, Ruys AJ. Reporting for Duty: The duty cycle in Functional Electrical Stimulation (FES) research - Part I: Critical commentaries of the literature. Eur J Transl Myol 2018;28: In press.

55. Ekelem A, Goldfarb M. Supplemental stimulation improves swing phase kinematics during exoskeleton assisted gait of SCI subjects with severe muscle spasticity. Front Neurosci 2018 Jun 1;12:374. doi: 10.3389/fnins.2018.00374. eCollection 2018.

56. Angeli CA, Boakye M, Morton RA, et al. Recovery of Over-Ground Walking after Chronic Motor Complete Spinal Cord Injury. N Engl J Med 2018;379(13):1244-50. doi: 10.1056/NEJMoa 1803588. Epub 2018 Sep 24.

57. Harkema SJ, Legg Ditterline B, Wang S, et al. Epidural Spinal Cord Stimulation Training and Sustained Recovery of Cardiovascular Function in Individuals With Chronic Cervical Spinal Cord Injury. JAMA Neurol 2018 Sep 17. doi: 
10.1001/jamaneurol.2018.2617. [Epub ahead of print].

58. Herrity AN, Williams CS, Angeli CA, et al. Lumbosacral spinal cord epidural stimulation improves voiding function after human spinal cord injury. Sci Rep 2018 Jun 6;8(1):8688. doi: 10.1038/s41598-018-26602-2.

59. Aslan SC, Legg Ditterline BE, Park MC, et al. Epidural Spinal Cord Stimulation of Lumbosacral Networks Modulates Arterial Blood Pressure in Individuals With Spinal Cord Injury-Induced Cardiovascular Deficits. Front Physiol 2018;9:565. doi: 10.3389/fphys.2018.00565. eCollection 2018.

60. Harkema SJ, Wang S, Angeli CA, et al. Normalization of Blood Pressure With Spinal Cord Epidural Stimulation After Severe Spinal Cord Injury. Front Hum Neurosci 2018;12:83. doi: 10.3389/fnhum.2018.00083. eCollection 2018.

61. Terson de Paleville DGL, Harkema SJ, Angeli CA. Epidural stimulation with locomotor training improves body composition in individuals with cervical or upper thoracic motor complete spinal cord injury: A series of case studies. J Spinal Cord Med 2018 Mar 14:1-7.doi: 10.1080/10790268. 2018.1449373. [Epub ahead of print]

62. Hubscher $\mathrm{CH}$, Herrity AN, Williams CS, et al. Improvements in bladder, bowel and sexual outcomes following task-specific locomotor training in human spinal cord injury. PLoS One 2018;13(1):e0190998. doi: 10.1371/journal.pone. 0190998. eCollection 2018.
63. Wagner FB, Mignardot JB, Le Goff-Mignardot CG, et al. Targeted neurotechnology restores walking in humans with spinal cord injury. Nature 2018 Nov;563(7729):65-71. doi: 10.1038/s41586-0180649-2. Epub 2018 Oct 31.

64. Formento E, Minassian K, Wagner F, et al. Electrical spinal cord stimulation must preserve proprioception to enable locomotion in humans with spinal cord injury. Nat Neurosci 2018 Oct 31. doi: 10.1038/s41593-018-0262-6. [Epub ahead of print]

65. Capogrosso M, Wagner FB, Gandar J, et al. Configuration of electrical spinal cord stimulation through real-time processing of gait kinematics. Nat Protoc 2018Sep;13:2031-61. doi: 10.1038/s41596018-0030-9.

66. Capogrosso M, Gandar J, Greiner N, et al. Advantages of soft subdural implants for the delivery of electrochemical neuromodulation therapies to the spinal cord. J Neural Eng 2018;15:026024. doi: 10.1088/1741-2552/aaa87a. 10.1088/1741-2552/aaa87a.

67. Moraud EM, von Zitzewitz J, Miehlbradt J, et al. Closed-loop control of trunk posture improves locomotion through the regulation of leg proprioceptive feedback after spinal cord injury. Sci Rep 2018;8:76. doi: 10.1038/s41598-017-18293-y.

Received for publication: 02/8/2018

Revision received: 20/9/2018

Accepted for publication: 21/9/2018 